\title{
Ce que tout enseignant devrait savoir concernant le raisonnement clinique*
}

\author{
What every teacher needs to know about clinical reasoning
}

Kevin W. EVA

\begin{abstract}
Résumé Contexte : Une des tâches principales de l'enseignant clinicien est d'aider les étudiants à faire le tri parmi les données du malade et à poser un diagnostic exact, tout en ayant comme objectif ultime un traitement approprié. Depuis les 30 dernières années, il y a eu de nombreux débats pour savoir quel modèle décrit le mieux les processus mentaux utilisés par les cliniciens experts pour prendre des décisions diagnostiques. But : Le but du présent essai est de procéder à une revue des publications scientifiques consacrées à ce sujet afin de mieux renseigner les cliniciens qui enseignent au lit du malade ou en clinique. Les forces et faiblesses de différentes méthodes d'investigation du raisonnement clinique seront évaluées en utilisant comme cadre conceptuel une des typologies fondamentales qui distingue les modèles de raisonnement analytique (raisonnement conscient, contrôlé) par rapport aux modèles de raisonnement non-analytique (raisonnement inconscient, automatique). Discussion : Les résultats d'études récentes suggèrent que les enseignants cliniciens devraient promouvoir limportance des deux types de raisonnement, permettant ainsi aux étudiants de faire appel à des processus mentaux variés et appropriés au contexte. Des conseils pratiques sont formulés à l'intention des enseignants.
\end{abstract}

Mot clés Education médicale ; premier cycle/méthodes ; compétence clinique/éducation ; prise de décision ; raisonnement clinique; enseignement/méthodes; revue systématique.

Abstract Context: One of the core tasks assigned to clinical teachers is to enable students to sort through a cluster of features presented by a patient and accurately assign a diagnostic label, with the development of an appropriate treatment strategy being the end goal. Over the last 30 years there has been considerable debate within the health sciences education literature regarding the model that best describes how expert clinicians generate diagnostic decisions. Purpose: The purpose of this essay is to provide a review of the research literature on clinical reasoning for frontline clinical teachers. The strengths and weaknesses of different approaches to clinical reasoning will be examined using one of the core divides between various models [that of analytic (i.e. conscious/controlled) versus non-analytic (i.e. unconscious/automatic) reasoning strategies] as an orienting framework. Discussion: Recent work suggests that clinical teachers should stress the importance of both forms of reasoning, thereby enabling students to marshal reasoning processes in a flexible and context-specific manner. Specific implications are drawn from this overview for clinical teachers.

Key words Education medical; undergraduate/methods; clinical competence/education; decision making; clinical reasoning teaching/methods; review literature.

Pédagogie Médicale 2005 ; $6: 225-234$

* Traduit par Georges Bordage (Department of Medical Education - College of Medicine - University of Illinois at Chicago Etats-Unis), de "What every teacher needs to know about clinical reasoning " ${ }^{48}$ avec la permission de Medical Education et Blackwell Publishing Ltd.

1- Program for Educational Research and Development - Department of Clinical Epidemiology and Biostatistics - McMaster University Hamilton - Ontario L8N 3Z5- Canada

mailto : evakw@mcmaster.ca 


\section{Références}

A 2 heures du matin, un mari amène en salle d'urgence son épouse âgée de 43 ans parce qu'elle souffre d'une dyspnée aiguë l'ayant réveillée soudainement à 23 heures. Elle sest sentie nauséeuse et a vomi une petite quantité de bile. Elle s'est alors plainte de douleurs rétrosternales, exacerbées lors de la respiration profonde. Depuis quelques jours, elle a eu des petites expectorations sanguinolentes. Depuis 4 jours, elle ne se sentait pas bien, se plaignant d'un mal de gorge et d'une congestion sinusale qui se sont résorbés. Elle a eu des poussées de fière et des frissons à plusieurs reprises depuis quelques jours. La nuit précédente, elle s'est éveillée se sentant oppressée, mais le tout était rentré dans l'ordre en peu de temps. Elle a des antécédents de bronchite'.

Quel est le diagnostic le plus probable? A l'instar de la question " qui est le coupable? " dans un roman policier, le défi diagnostique consiste à considérer chaque élément d'information disponible et à trouver l'explication la plus plausible pour l'ensemble du tableau. La tâche n'est pas toujours facile. Elle consiste le plus souvent à bien observer, à faire une anamnèse appropriée et un examen physique précis, à générer des hypothèses diagnostiques, à évaluer le lien entre chaque élément d'information et chaque hypothèse et à tenter de confirmer ou réfuter les hypothèses en demandant des examens diagnostiques complémentaires appropriés. Contrairement au lecteur du roman policier, le clinicien doit aussi souvent déterminer si une nouvelle information doit être recueillie et, le cas échéant, quand et comment.

Le défi, pour l'enseignant clinicien, est sans doute encore plus redoutable. Non seulement l'enseignant clinicien doit être capable d'exécuter toutes les tâches mentionnées ci-dessus mais doit aussi trouver une façon de transmettre à l'apprenti-diagnosticien ses connaissances et sa démarche (son raisonnement), afin de faciliter l'apprentissage de l'étudiant. Depuis les 50 dernières années, il est devenu clair que la capacité d'enseigner la démarche clinique est à la fois liée au savoir médical de l'enseignant concernant la matière à enseigner (expertise de contenu) mais aussi distincte de celui-ci ${ }^{2}$. L’entretien de la capacité à enseigner en milieu clinique requiert, entre autre, une compréhension des stratégies de raisonnement que les cliniciens experts utilisent, souvent de façon inconsciente, afin de décortiquer un problème diagnostique comme celui présenté en introduc$\operatorname{tion}^{3}$. Le fait que les mécanismes psychologiques sousjacents liés à de telles stratégies mentales ne soient pas toujours accessibles à l'introspection ${ }^{4}$ pose un défi additionnel à l'enseignant.

Le but du présent essai est de présenter aux enseignants cliniciens une revue synthétique des publications scientifiques consacrées au raisonnement clinique. Cependant, peu de détails seront présentés concernant les méthodes d'investigation qui ont conduit à chaque conclusion présentée ; des références sont incluses à cette fin pour les lecteurs intéressés. L'accent sera davantage mis sur ce qu'on comprend aujourd'hui du cheminement qu'utilisent les cliniciens pour résoudre les défis diagnostiques et sur les conséquences pédagogiques qui en découlent. La discussion portera sur les techniques pédagogiques susceptibles d'accroître la probabilité que les étudiants développent les apprentissages nécessaires pour devenir de bons diagnosticiens et sur les stratégies nécessaires pour évaluer de façon juste l'acquisition des compétences voulues. Examinons d'abord de plus près comment résoudre le problème posé au point de départ.

\section{Quel est le diagnostic le plus probable (et comment y arriver)?}

De façon traditionnelle, les pédagogues médicaux ont étudié le raisonnement clinique selon un modèle analytique. Ce modèle prend pour acquis que l'expertise clinique repose sur une analyse rigoureuse et attentive de la relation entre les signes, les symptômes et les hypothèses diagnostiques. Par exemple, dans le traité de référence « Principes de médecine interne " d'Harrison, la dyspnée est présentée comme un indice à la fois de pneumonie et d'embolie pulmonaire. Dans le cas présent, les données additionnelles de maux de gorge, nausées et vomissements suggèrent davantage une pneumonie ${ }^{5}$, alors que les expectorations sanguinolentes suggèrent une embolie pulmonaire ${ }^{6}$. Dans les deux hypothèses, il est tenu pour acquis que les données clés sont clairement évidentes et que le raisonnement clinique consiste à comprendre le lien entre les données et les maladies sous-jacentes. La formulation d'une liste d'hypothèses diagnostiques pertinentes (un diagnostic différentiel) et l'utilisation d'un algorithme diagnostique approprié permettront alors de soupeser chaque hypothèse selon sa probabilité relative ${ }^{6}$.

Ce genre de raisonnement est notamment bien représenté par des modèles de raisonnement qui s'appuient sur le théorème de Bayes ou sur des analyses de régression ${ }^{7,8}$. En bref, ces modèles postulent que le médecin connait la 
probabilité a priori du diagnostic en question et la probabilité conditionnelle de chaque donnée clinique (signes, symptômes, examens complémentaires) liée à chaque diagnostic. Le modèle mathématique sous-jacent permet de calculer la probabilité a posteriori de chaque diagnostic considéré. Ce processus, illustré dans la figure 1, est utilisé par ceux qui font la promotion de la médecine fondée sur les preuves, ${ }^{90}$. Alors que certains prétendent que le traitement des informations par " chaînage avant " (qui consiste à raisonner à partir des données vers un diagnostic, en anglais forward reasoning), illustré sur la figure 1, rend compte de façon privilégiée de l'essence du raisonnement clinique de l'expert ${ }^{11,12}$, les probabilités a posteriori ainsi obtenues peuvent tout aussi bien être utilisées pour guider l'acquisition et l'analyse de données additionnelles. Le modèle reste cependant de nature analytique, dès lors qu'une analyse rigoureuse et attentive se poursuit.

De fait, des processus analytiques ont été utilisés de diverses façons dans différents modèles d'expertise ${ }^{13-15}$. Au coeur de chaque approche toutefois, réside le postulat fondamental que des règles causales, liant données (signes et symptômes) et catégories (diagnostic), peuvent être inférées, de sorte que le développement de l'expertise en raisonnement clinique consiste à développer et élaborer des règles qui deviennent de plus en plus en accord avec la réalité. Selon cette approche, le rôle de l'enseignant est de faciliter le développement de ces règles. Par exemple, Elieson et Papa ont démontré les bénéfices pédagogiques liés au fait de fournir aux étudiants des aides diagnostiques qui décrivent de façon explicite les relations probabilistes entre les données ${ }^{16}$. Toutefois, il n'est pas nécessaire de partager une vision aussi radicale de l'utilité de probabilités aussi explicites pour exploiter pédagogiquement la connaissance du processus du raisonnement analytique dans son enseignement; l'enseignant clinicien fait la promotion de théories intuitives de la valeur du raisonnement analytique à chaque fois qu'il propose aux étudiants d'être " objectif " et de " considérer soigneusement toutes les données disponibles avant de générer des hypothèses diagnostiques ".

Il y a cependant une autre façon de résoudre le problème posé, qui a reçu de plus en plus d'attention depuis une quinzaine d'années. Par exemple, tentez de poser un diagnostic à partir du cas suivant.

A 2 heures du matin, un mari amène en salle d'urgence son épouse àgée de 43 ans parce qu'elle souffre d'une dyspnée aigue l'ayant réveillée soudainement à 23 heures. Elle s'est sentie nauséeuse et a vomi une petite quantité de bile. Elle s'est alors plainte de douleurs rétrosternales, exacerbées lors de la respiration profonde. Depuis quelques jours, elle a eu des petites expectorations sanguinolentes. Depuis 4 jours, elle ne se sentait pas bien, se plaignant d'un mal de gorge et d'une congestion sinusale qui se sont résorbés. Elle a eu des poussées de fièvre et des frissons à plusieurs reprises depuis quelques jours.

\section{Figure 1 :}

\section{Raisonnement clinique selon une approche analytique}

\section{Données initiales} $\mathrm{w}(\mathrm{A}, 1)$

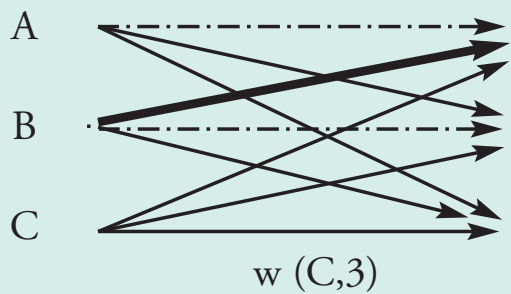

\section{Hypothèses \\ diagnostiques}

\section{Probabilité a posteriori}

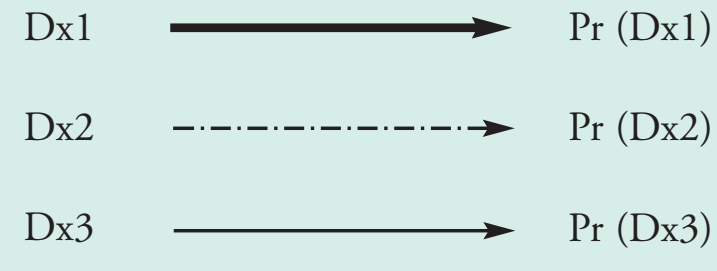

Chaque diagnostic est représenté par un chiffre et chaque donnée par une lettre. Chaque donnée maintient une relation unique (un poids) avec chaque diagnostic. La taille de chaque poids est indiquée par la dimension de la flèche; deux ont été étiquetées (par exemple, le poids de la relation entre la donnée A et le diagnostic 1). En combinant les probabilités a priori et les poids des données on obtient la probabilité a posteriori (Pr) pour chaque hypothèse diagnostique tout en tenant compte de l'ensemble des données observées. 


\section{Références}

La nuit précédente, elle s'est éveillée se sentant oppressée, mais le tout était rentré dans l'ordre en peu de temps. Elle a des antécédents de bronchitel.

Même un lecteur peu expérimenté en médecine reconnâ̂tra que le diagnostic différentiel comporte une pneumonie et une embolie pulmonaire. Pourquoi ? Parce que ce cas a déjà été vu (au début de l'article). Si l'on vous demande : "Divisez 120 par 10?", la plupart d'entre vous peuvent répondre « 12 » rapidement et sans effort. De la même façon, les cliniciens sont souvent dans une situation similaire, où ils n'ont pas besoin de faire l'effort de " raisonner ». Il s'agit de reconnaitre une "forme ", une configuration (de l'anglais, "pattern recognition"); ce type de processus est l'exemple même d'un raisonnement nonanalytique, illustré sur la figure 2. Il s'agit essentiellement de comparer le cas présent à des cas vus antérieurement et d'utiliser ces expériences passées pour poser des jugements concernant la probabilité qu'un cas particulier donné fait partie d'une catégorie diagnostique particulière ${ }^{17,18}$. L'exemple utilisé ici en est une caricature, dans la mesure où toutes les informations ont été présentées de façon strictement identiques dans les deux énoncés; mais, comme l'illustre la figure 2, il n'est pas nécessaire que toutes les données soient exactement semblables pour que l'on puisse identifier une correspondance convenable.

Ce genre de " raisonnement » est possible, avec suffisamment d'automaticité, pour avoir souvent lieu sans aucun effort conscient apparent. Malgré la tendance que nous avons, en tant qu'humains, à proposer des explications pour nos actions, en réalité la source de nos actions et de nos décisions nous est souvent inconnue ${ }^{4}$. De ce fait, bien qu'il soit impossible de vérifier si les réponses d'un clinicien sont valides lorsqu'on lui demande simplement s'il a utilisé une reconnaissance des formes ("pattern recognition "), il est indéniable que les cliniciens utilisent des processus non analytiques lorsqu'ils prennent des décisions diagnostiques ${ }^{19}$. Par exemple, dans une série d'études scientifiques, Brooks et collaborateurs ont démontré que la justesse du diagnostic est plus élevée lorsque les cas sont semblables à ceux vus antérieurement que lorsqu'ils en sont très différents sur le plan perceptuel $^{20}$. De plus, Hatala et collaborateurs ont établi que même des données non pertinentes (comme le fait d'être un banquier) ont un impact sur le diagnostic de cas subséquents pour lesquels la donnée non pertinente est similaire $^{21}$.

Pour certains, la capacité d'utiliser des bases non analytiques pour prendre des décisions cliniques augmente avec l'expérience et, par conséquent, le recours à des processus de reconnaissance de formes ("patterns") ne devrait pas être conseillé aux étudiants en formation par peur de "conséquences graves potentielles $»^{22}$. A l'extrême, des purs novices, qui n'ont pas d'expériences antérieures sur lesquelles ils peuvent compter, seront par conséquent incapables d'utiliser un raisonnement par similitude. En réalité toutefois, il a été démontré que la stratégie utilisée par des

Figure 2 :

Raisonnement clinique selon une approche non analytique

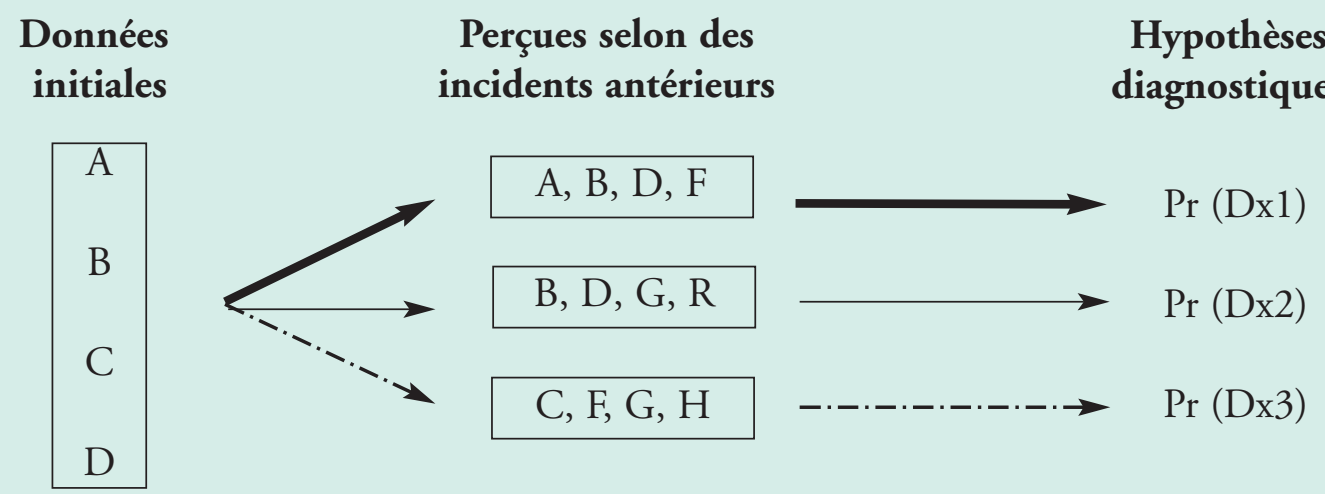

Chaque diagnostic est représenté par un chiffre et chaque donnée par une lettre. Chaque malade (représenté par un rectangle) se présente avec un ensemble de données. Cet ensemble est comparé (de façon inconsciente) à des exemples vus antérieurement, résultant ainsi en une probabilité pour chaque hypothèse. La taille de l'association entre le malade en question et les expériences antérieures est variable, telle qu'indiquée par la dimension des flèches. 
étudiants en médecine, même très juniors, est qualitativement semblable à celle utilisée par des cliniciens d'expérience; les uns et les autres génèrent des hypothèses très tôt, vraisemblablement en faisant appel, de façon non analytique, à des expériences antérieures ${ }^{23}$. Les cliniciens d'expérience sont plus susceptibles de générer le bon diagnostic parce que, de façon prévisible, ils disposent d'une base de données plus vaste à laquelle ils peuvent faire appel. De façon concrète, chaque fois que l'on a explicitement comparé les conséquences d'un enseignement du raisonnement se référant respectivement soit à une approche analytique, soit à la confiance en son jugement clinique non analytique, la justesse diagnostique a été au moins aussi bonne, sinon meilleure, pour les étudiants formés au raisonnement non analytique, et ce même pour des jeunes novices ${ }^{22,32}$. Des bases non analytiques ne sont pas moins bonnes que des formes plus analytiques de raisonnement. Ainsi les enseignants cliniciens devraient indiquer à leurs étudiants que la ressemblance (similitude) du cas présent à des cas antérieurs peut servir de guide utile. L'éventualité de « conséquences graves » est un argument spécieux étant donné que la responsabilité finale des soins aux malades est assurée précisément par l'enseignant clinicien et non par l'étudiant, le novice.

\section{L'association de stratégies analytiques et non analytiques}

Ceci dit, il semble établi que trop se fier à des approches non analytiques peut être une cause d'erreur diagnostique. Les impressions premières, bien qu'utiles, sont fréquem- ment erronées, même chez des cliniciens d'expérience ${ }^{24}$. Des éléments contextuels, tel que le fait de recevoir des suggestions diagnostiques, peuvent diminuer à la fois la probabilité d'identifier des données propres à certains diagnostics ${ }^{25}$ et celle d'attribuer correctement un poids relatif aux données cliniques en lien avec les hypothèses alternatives $^{26}$. Eva a démontré que ces biais proviennent d'une confiance excessive envers des processus non analytiques. La simple directive de dresser une liste explicite des données objectives contenues dans le cas (c'est-à-dire de promouvoir des processus davantage analytiques) est suffisante pour éliminer ce genre de biais ${ }^{27}$. Il est essentiel toutefois que la composante analytique de la démarche ait lieu de façon très rapprochée par rapport au processus réel de jugement diagnostique.

Qu'en est-il pour l'enseignant clinicien ? D'abord, il est important de reconnaitre que ces deux formes de processus mentaux ne sont pas mutuellement exclusives. Il est fort probable qu'elles contribuent l'une et l'autre à la décision finale concernant chaque cas clinique (autant pour les novices que pour les experts). La reconnaissance d'une similitude provoquera, dans certains cas, une démarche analytique pour le cas en cours qui sera analogue aux analyses faites lors d'un cas similaire antérieur. Par conséquent, la forme optimale de raisonnement clinique doit être vue comme un modèle mixte où, à la fois, des processus analytiques et non-analytiques sont mobilisés. Un tel modèle est présenté sur la figure 3. Dans ce modèle, le clinicien se fait une représentation mentale du problème au moment de rencontrer le malade. Cette représentation mentale conduit à la vérification d'hypothèses lors de

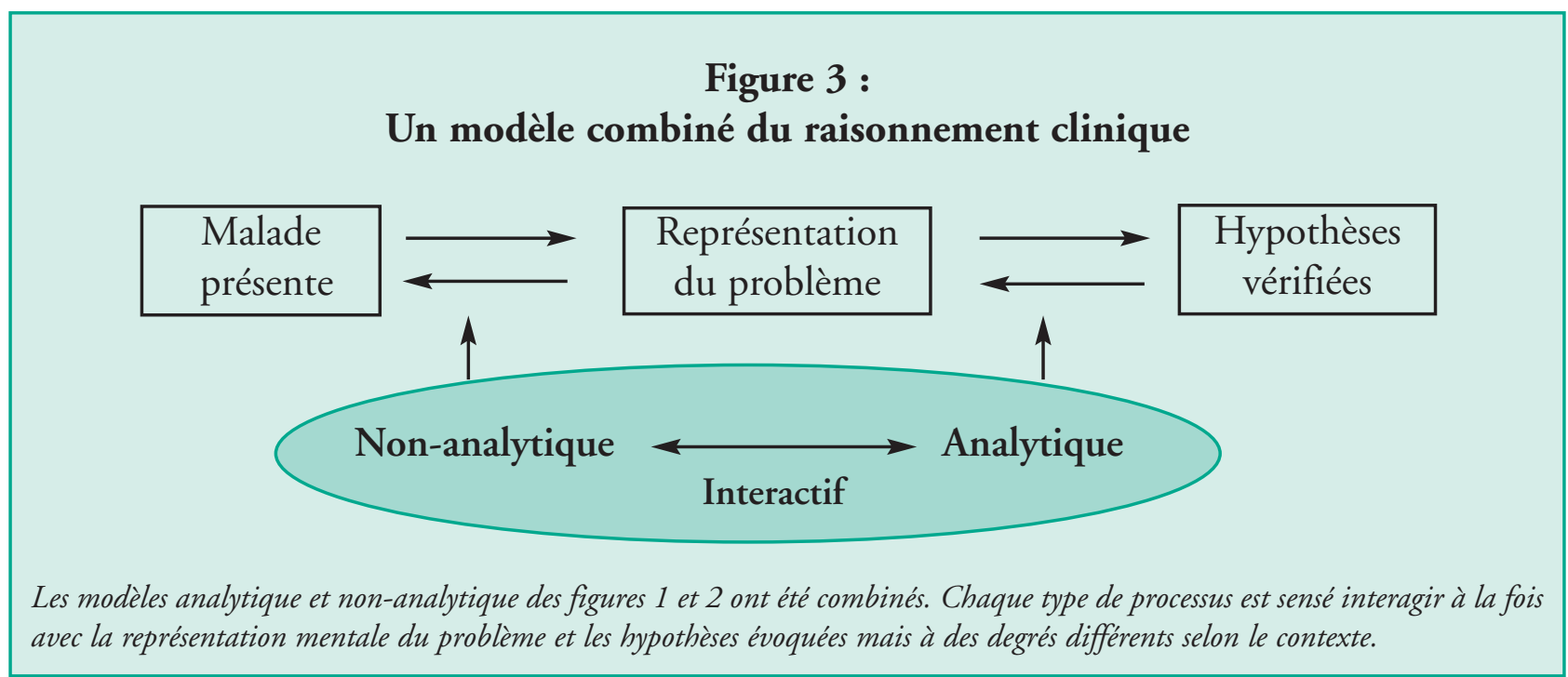




\section{Références}

l'anamnèse, de l'examen physique et de la demande d'examens diagnostiques complémentaires. Il est important de noter l'aspect bidirectionnel du raisonnement. Le résultat de la vérification d'hypothèses influencera la représentation mentale que se fait le clinicien du problème et cette représentation mentale pourra à son tour influencer la façon dont le problème est perçu. Ce flux bidirectionnel peut être présent à la fois chez les novices et les experts ${ }^{28}$. De plus, il est important de noter que, même si l'on peut suspecter que des processus non analytiques soient majoritaires pendant la phase initiale du raisonnement face à un nouveau cas et qu'à l'inverse des processus analytiques soient dominants au moment de vérifier les hypothèses, ces deux formes de raisonnement doivent être considérées comme très interactives. Ces mécanismes sont complémentaires, davantage qu'ils ne s'inscrivent dans un continuum, chacun influençant l'autre et contribuant à la justesse du raisonnement ${ }^{29-31}$.

Des travaux de recherche récents accréditent concrètement ce modèle ${ }^{32}$. Lors d'un enseignement à de purs novices (des étudiants de premier cycle en psychologie) ayant trait au diagnostic des électrocardiogrammes, certains ont reçu des consignes leur indiquant de se fier à leur sens de similitude (une base non-analytique du raisonnement clinique) au moment de pratiquer leur démarche diagnostique et d'être évalués, mais d'éviter de sauter trop vite sur une conclusion en considérant, de façon explicite, chaque donnée présente sur l'électrocardiogramme (une stratégie analytique). Ce groupe a eu de meilleurs résultats que deux autres groupes où les participants ont reçu respectivement et exclusivement des consignes soit non analytiques, soit analytiques. La performance diagnostique de ces deux derniers groupes a été semblable. Ces résultats confirment et prolongent ceux de travaux antérieurs où des consignes combinées (analytiques et non analytiques) produisaient une meilleure justesse diagnostique que des consignes purement analytiques ${ }^{33}$. Lors de ces deux études, les étudiants qui n'avaient reçu que des consignes analytiques se trouvèrent confrontés à un véritable déluge de données cliniques, qui compliquait l'établissement de concordances de formes ("pattern") avec une seule entité diagnostique. De plus, d'autres travaux de recherche suggèrent que l'absence de processus analytique pour confirmer une hypothèse peut aboutir à une fermeture prématurée du raisonnement et à des erreurs diagnostiques, même chez des cliniciens très expéri- mentés ${ }^{24}$. Afin d'éviter l'une ou l'autre de ces éventualités, les enseignants cliniciens devraient promouvoir les deux formes de raisonnement en combinaison. Une présentation plus détaillée des conséquences de cette recommandation sera faite après un bref exposé concernant la stabilité de certaines stratégies diagnostiques.

\section{L'état de l'art}

Compte tenu des descriptions faites à ce jour du raisonnement clinique, on pourrait déduire qu'il est nécessaire que l'enseignant clinicien aide les étudiants à devenir des bons "solutionneurs de problème " ou des bons " coordonnateurs de processus analytiques et non-analytiques ». Une telle conception générique du processus diagnostique est dépassée et inexacte parce quili a été démontré à maintes reprises que la résolution d'un problème particulier n'est pas un bon prédicteur de la capacité de résoudre d'autres problèmes (ce dont rend compte le concept dénommé "spécificité de cas "; de l'anglais "case specificity »), même au sein d'un même domaine de spécialisation d'exercice ou de spécialitéé ${ }^{34,35}$. La capacité de raisonner n'est pas un « trait " propre à un individu. Sans doute, certains individus sont de meilleurs diagnosticiens que d'autres mais l'enseignant clinicien doit savoir reconnaître que le contexte dans lequel un problème se situe joue un rôle majeur sur la justesse de la décision prise et sur l'équilibre optimal entre plusieurs "stratégies " cognitives potentielles. Le « contexte» comprend à la fois des facteurs situationnels (par exemple, le milieu clinique et les cas vus récemment) et des facteurs personnels (par exemple, le niveau d'expérience du clinicien et son état de pensée, son opinion du moment).

Malgré tous les efforts faits pour offrir un programme de formation uniforme à tous, les étudiants n'ont pas tous les mêmes expériences. Différents étudiants voient différents malades, prennent en compte différents aspects de chaque cas vu et tirent différentes leçons de leurs réflexions. Chacune de ces différences aura un effet sur la façon dont chaque étudiant abordera un cas donné (c'est-à-dire que des éléments circonstanciels spécifiques influenceront la "stratégie de raisonnement " adoptée). Cette conception circonstancielle de la clinique constitue sans doute la raison fondamentale qui conduit à souhaiter offrir aux étudiants des stratégies multiples qui leur permettront de résoudre un problème clinique. Dans certains cas, l'utilisation intensive de reconnaissance des formes ("pattern recognition ") sera la plus susceptible de révéler la bonne solution. Dans d'autres 
cas, une anamnèse plus complète, l'utilisation d'un algorithme diagnostique ou une approche physiopathologique pourraient être requises. Plus le clinicien aura d'outils dans son coffre à outils, plus certains outils seront susceptibles de faciliter l'exécution de la tâche requise.

Ainsi, il est de plus en plus clair qu'aucun modèle ou conception unique du raisonnement ne pourra rendre compte de façon complète de la notion d' " expert » dans un domaine aussi vaste que le raisonnement clinique. Par analogie, considérez les habiletés requises pour lire. Afin d'analyser les éléments requis pour lire, Levy et Hinchley ont administré une série de 11 tests et ont trouvé que bien que les lecteurs les plus faibles soient en moyenne moins performants que les bons lecteurs, chaque lecteur, pris individuellement, avait ses forces et ses faiblesses ${ }^{36}$. Ainsi, $56 \%$ des bons lecteurs firent preuve de déficits lors d'un ou plusieurs tests et $58 \%$ des lecteurs faibles firent preuve de capacités supérieures lors d'un ou plusieurs tests. Ainsi, il est probable que l'expertise en raisonnement diagnostique devrait être considérée comme une entité malléable qui permet aux cliniciens compétents de compenser certaines faiblesses face à certains cas donnés. Ceci permet au clinicien de s'adapter aux exigences (circonstances) de la situation en utilisant de façon flexible (bien que souvent de façon inconsciente) une panoplie de processus.

\section{Conséquences pour l'enseignant clinicien}

Au total, il y a de nombreux débats en pédagogie médicale concernant la structure de l'expertise en médecine. Vers la fin des années 70, Elstein et collaborateurs ont proposé le modèle hypothético-déductif du raisonnement, qui postulait en l'occurrence que, confrontés à un nouveau cas de malade, les médecins génèrent un ensemble d'hypothèses qu'ils testent ensuite avec des données présentes ${ }^{37}$. Depuis, plusieurs cadres conceptuels ont été évoqués concernant l'organisation des connaissances en mémoire et les recherches effectuées depuis une quinzaine d'années suggèrent qu'il n'existe pas un cadre explicatif unique ${ }^{38}$. Les travaux de recherche récemment accumulés suggèrent de développer l'enseignement clinique selon une approche plus éclectique, qui reconnaisse à la fois les bénéfices d'approches analytiques et non-analytiques, permettant ainsi aux étudiants de tirer avantage du meilleur des deux mondes $^{32}$. Le rôle prépondérant que joue le contexte dans lequel le problème survient exige que l'on présente aux étudiants une variété de stratégies qui leur donneront suffi- samment de flexibilité pour répondre aux multiples exigences de la situation. Avec ces considérations et cette conceptualisation du raisonnement comme toile de fond, comment peut-on évaluer nos méthodes pédagogiques courantes et stimuler le développement de nouvelles approches?

D'abord et avant tout, cette synthèse souligne l'importance d'enseigner à partir d'exemples. Plus les étudiants auront l'occasion de se bâtir très tôt un répertoire de cas cliniques, plus rapidement ils se bâtiront des assises solides à partir desquelles les processus non analytiques pourront s'alimenter. Cette idée n'est ni nouvelle, ni spécifique à la pédagogie médicale ; il s'agit d'un principe fondamental de tout bon enseignement. En revanche, ce qui est relativement nouveau est le fait qu'un nombre limité d'exemples complexes et élaborés est vraisemblablement insuffisant comme moyen d'enseignement efficace. La spécificité du contexte et le besoin de se bâtir une base de données (un répertoire de problèmes), à partir de laquelle un raisonnement analogique peut être développé, exigent que les étudiants soient exposés à beaucoup d'exemples (cas, malades), qu'ils soient activement impliqués dans le processus de résolution de problème et que les exemples constituent un éventail représentatif de la variété de différentes présentations possibles d'une entité pathologique ${ }^{34}$. Ce dernier critère est de plus en plus important étant donné que plusieurs systèmes de santé à travers le monde limitent la chance que les étudiants ont de rencontrer de façon aléatoire certaines conditions médicales lors de leurs stages en milieu clinique. Par conséquent, les enseignants et les directeurs de programmes doivent être conscients de ce fait et doivent faire preuve de créativité afin de s'assurer que leurs étudiants verront un éventail adéquat de malades, à la fois en quantité et en qualité, qui sera pédagogiquement utile.

Deuxièmement, les enseignants cliniciens doivent reconnaître que les programmes traditionnels en deux étapes, remontant à Flexner ${ }^{39}$, où les étudiants s'approprient d'abord les sciences de base avant d'être exposés à des problèmes cliniques, sont vraisemblablement révolus. Des travaux de recherche en pédagogie médicale suggèrent que la compréhension de mécanismes fondamentaux peut aider le clinicien à générer des hypothèses diagnostiques justes et par conséquent doit continuer à faire partie du programme de formation ${ }^{40,41}$. Toutefois, il est important de reconnaitre que cette stratégie ne représente qu'une façon de parvenir au bon diagnostic, et non la seule façon. Symétriquement, le fait de n'être 


\section{Références}

exposé exclusivement qu’à des malades en contexte clinique, sans mettre en exergue les mécanismes physiopathologiques sous-jacents, serait rendre de mauvais services aux étudiants en diminuant leur chance d'explorer des pistes pertinentes de solutions lors de problèmes cliniques ultérieurs.

Troisièmement, l'exploitation des problèmes cliniques doit refléter l'utilisation prévisible des connaissances qui en résulteront. Les cliniciens rencontrent rarement des nouveaux cas dont le diagnostic est connu. Le fait d'étudier certains cas dans un volume de référence où l'étudiant connaît déjà le diagnostic en faisant le lien avec le titre du chapitre (ou le sujet de la leçon magistrale) ne garantit pas nécessairement que l'étudiant sera capable de reconnaître un cas semblable en clinique. Si le malade et la représentation du cas de la figure 3 sont complètement imbriqués dans un diagnostic particulier, l'opportunité de développer la phase de vérification d'hypothèses est perdue. De fait, plusieurs chercheurs ont démontré les avantages pédagogiques d'exploiter des recrutements non sélectionnés (" pratiques mixtes »), permettant aux étudiants d'être exposés à des situations ressortissant de plusieurs disciplines cliniques, plutôt que procéder en les exposant successivement à des «blocs » de situations du même champ clinique ${ }^{42,43}$.

De plus, l'enseignant clinicien ne doit pas se fier aux étudiants pour faire spontanément des comparaisons significatives entre différents problèmes. Les étudiants sont davantage susceptibles de raisonner par analogie lorsqu'ils ont été incités à identifier de façon explicite les similitudes entre concepts sous-jacents présents respectivement dans des problèmes apparemment différents ${ }^{44}$. Ainsi et autant que possible, des liens explicites doivent être développés entre les concepts et principes concernés par l'analyse d'un exemple clinique et ceux liés à des exemples antérieurs. Outre les bénéfices propres à cette stratégie pédagogique, l'enseignant clinicien pourra aussi mieux identifier la nature des difficultés de leurs étudiants. Il est bien reconnu que les experts ont des difficultés à prédire les erreurs des autres ${ }^{45}$. En donnant l'occasion aux étudiants de révéler leurs erreurs idiosyncrasiques, les enseignants cliniciens pourront davantage cibler leur enseignement sur des aspects dont leurs étudiants risquent de tirer le meilleur profit.

Enfin, la flexibilité du raisonnement clinique et l'importance du contexte ont des conséquences très importantes pour l'évaluation des étudiants. L'enseignant ne doit pas prendre pour acquis que l'étudiant a compris la physio-pathologie sous-jacente simplement parce que son diagnostic ou son traitement sont justes. A l'inverse, même si l'étudiant peut expliquer les mécanismes physiopathologiques, il n'est pas garanti qu'il pourra poser un diagnostic juste en situation clinique ultérieure. Dans des domaines où la spécificité du contexte joue un rôle important (c'est-à-dire tous les domaines), des " biopsies multiples » sont nécessaires pour évaluer, de façon juste, la performance des étudiants. Alors qu'il paraitrait impensable d'évaluer les connaissances d'un individu avec une seule question à choix de réponses, il est moins spontanément admis qu'il faille recourir à un échantillon suffisamment large de situations pour évaluer d'autres aspects de la compétence. Toutefois, il est clair que la spécificité du contexte (de cas) est la cause principale de la piètre qualité psychométrique de certaines méthodes d'évaluations, comme les épreuves de type "triple-saut ", les "PMPs» (de l'anglais, "Patient Management Problems»), les examens écrits à réponse longue et les examens oraux ${ }^{46}$. Plus il faut de temps aux étudiants pour accomplir une tâche d'évaluation, plus on diminue le nombre d'observations permettant de les évaluer à plusieurs reprises. Afin d'assurer la fidélité des notes obtenues, l'enseignant clinicien doit utiliser des méthodes d'évaluation grâce auxquelles il est possible de documenter les comportements et les apprentissages qu'ils sous-tendent à plusieurs reprises, par exemple avec des Examens cliniques objectifs structurés (ECOS), des exercices de raisonnement clinique et des examens à choix de réponses ${ }^{47}$.

\section{Remerciements de l'auteur}

L'auteur (Kevin Eva) désire remercier John Bligh pour l'avoir motivé à rédiger cet essai, ainsi que Lee Brooks, Geoff Norman et Glenn Regehr pour leurs commentaires perspicaces lors de la préparation du manuscrit. Le contenu de cet essai a été présenté en partie en mai 2004 lors d'un atelier de formation à l'Association of Medical School Microbiology and Immunology Chairs. Contrat de recherche: aucun. Approbation éthique : non requise.

\section{Remerciements du traducteur}

Je suis également très reconnaissant envers John Bligh, rédacteur en chef de la revue Medical Education, et la maison d'édition Blackwell Publishing Ltd pour leur permission de traduire et publier l'article original. Je suis aussi très reconnaissant envers Kevin Eva pour avoir écrit la version princeps de cet essai. Enfin, je tiens à remercier vivement Jean Jouquan, Bernard Charlin et Mathieu Nendaz pour leurs précieux conseils lors de la révision de ma traduction du texte anglais. Je suis toutefois seul responsable de la version finale. 


\section{Références}

1. Cunnington JPW, Turnbull JM, Regehr G, Marriott M, Norman GR. The effect of presentation order in clinical decision making. Acad Med 1997;72(Suppl):40-2.

2. Wilkerson L, Irby DM. Strategies for improving teaching practices: a comprehensive approach to faculty development. Acad Med 1998;73:387-96.

3. Irby DM. What clinical teachers in medicine need to know. Acad Med 1994;69:333-42.

4. Bargh JA, Chartrand TL. The unbearable automaticity of being. Am Psychol 1999;54:462-79.

5. Levison ME. Pneumonia, including necrotising pulmonary infections (lung abscess). In: Braunwald E, Hauser $S L$, Fauci AS, Longo DL, Kasper DL, Jameson JL (eds). Harrison's Principles of Internal Medicine. 15th edn. New York : McGraw-Hill 2001;1475-85.

6. Goldhaber SZ. Pulmonary thromboembolism. In: Braunwald E, Hauser SL, Fauci AS, Longo DL, Kasper $D L$, Jameson JL (eds). Harrison's Principles of Internal Medicine. 15th edn. New York: McGraw-Hill 2001; 1508-13.

7. Weinstein MD, Fineburg HU, Elstein AS et al. Clinical Decision Analysis. Philadelphia: WB Saunders 1980.

8. Wigton RS. Use of linear models to analyse physicians' decisions. Med Decis Making 1988;8:241-52.

9. Sackett DL, Haynes RB, Guyatt GH, Tugwell P. Clinical Epidemiology: A Basic Science for Clinical Medicine. 2nd edn. Toronto: Little Brown 1991.

10. Fletcher $R$, Fletcher $S$, Wagner E. Clinical Epidemiology: The Essentials. 2nd edn. Baltimore: Williams \& Wilkins, 1996.

11. Patel VL, Goren GJ. Knowledge-based solution strategies in medical reasoning. Cognit Sci 1986;10:91-116.

12. Patel VL, Arocha JF, Kaufman DR. Diagnostic reasoning and medical expertise. Psychol Reason Motiv 1994;31:187-252.

13. Genero N, Cantor N. Exemplar prototypes and clinical diagnosis: toward a cognitive economy. J Soc Clin Psychol 1987;59-78.
14. Bordage G. Elaborated knowledge: a key to successful diagnostic thinking. Acad Med 1994;69:883-5.

15. Mandin H, Jones A, Woloschuk W, Harasym P. Helping students learn to think like experts when solving clinical problems. Acad Med 1997;72:173-9.

16. Elieson SW, Papa FJ. The effects of various knowledge formats on diagnostic performance. Acad Med 1994 ; 69(Suppl):81-3.

17. Brooks LR. Decentralised control in categorisation: the role of prior processing episodes. In: Neisser $U$ (ed.) Concepts and Conceptual Development: Ecological and Intellectual Factors in Categorisation. Cambridge: Cambridge University Press 1987;141-74.

18. Jacoby LL, Baker JG, Brooks LR. The priority of the specific: episodic effects in picture identification. J Exp Psychol Learn Mem Cogn 1989;15:275-81.

19. Norman GR, Brooks LR. The non-analytical basis of clinical reasoning. Adv Health Sci Educ 1997;2:17384.

20. Brooks LR, Allen SW, Norman GR. Role of specific similarity in a medical diagnostic task. J Exp Psychol Gen 1991;120:278-87.

21. Hatala RM, Norman GR, Brooks LR. Influence of a single example on subsequent electrocardiogram interpretation. Teach Learn Med 1999;11:110-7.

22. Coderre S, Mandin H, Harasym PH, Fick GH. Diagnostic reasoning strategies and diagnostic success. Med Educ 2003;37:695-703.

23. Neufeld VR, Norman GR, Feightner JW, Barrows HS. Clinical problem solving by medical students: a crosssectional and longitudinal analysis. Med Educ 1981; 15:315-22.

24. Eva KW. The ageing physician: changes in cognitive processing and their impact on medical practice. Acad Med 2002;77(Suppl):1-6.

25. Brooks LR, LeBlanc VR, Norman GR. On the difficulty of noticing obvious features in patient appearance. Psychol Sci 2000;11:112-7. 


\section{Références}

26. Eva $K W$, Brooks LR. The underweighting of implicitly generated diagnoses. Acad Med 2000;75(Suppl):81-3.

27. Eva KW. The influence of differentially processing information on diagnostic decision making. Dissertation. Hamilton (ON): McMaster University 2001.

28. Eva KW, Brooks LR, Norman GR. Forward reasoning as a hallmark of expertise in medicine: logical, psychological and phenomenological inconsistencies. In: Shohov SP (ed.) Advances in Psychological Research. Vol. 8. New York: Nova Science Publishers Inc. 2002;41-69.

29. Whittlesea BWA, Brooks LR, Westcott C. After the learning is over: factors controlling the selective application of general and particular knowledge. J Exp Psychol Learn Mem Cogn 1994;20:259-74.

30. Regehr G, Cline DJ, Norman GR, Brooks LR. Effect of processing strategy on diagnostic skill in dermatology. Acad Med 1994;69:S34-S36.

31. Kulatunga-Moruzi C, Brooks LR, Norman GR. Coordination of analytic and similarity-based processing strategies and expertise in dermatological diagnosis. Teach Learn Med 2001;13:110-6.

32. Ark T, Brooks LR, Eva KW. The best of both worlds: adoption of a combined (analytic and non-analytic) reasoning strategy improves diagnostic accuracy relative to either strategy in isolation. Presented at the annual meeting at the Association of American Medical Colleges; Boston (MA); November 2004.

33. Norman GR, Brooks LR, Colle CL, Hatala RM. The benefit of diagnostic hypotheses in clinical reasoning: experimental study of an instructional intervention for forward and backward reasoning. Cognit Instruct 2000;17:433-48.

34. Eva KW, Neville AJ, Norman GR. Exploring the aetiology of content specificity: factors influencing analogic transfer and problem solving. Acad Med 1998; 73(Suppl):1-5.

35. Eva KW. On the generality of specificity. Med Educ 2003;37:587-8.

36. Levy BA, Hinchley J. Individual and developmental differences in the acquisition of reading skills. In: Carr TH, Levy BA, eds. Reading and its Development: Component Skills Approaches. San Diego: Academic Press Inc. 1990;81-128.
37. Elstein AS, Shulman LS, Sprafka SA. Medical Problem Solving: An Analysis of Clinical Reasoning. Cambridge (MA): Harvard University Press 1978.

38. Custers EJFM, Regehr G, Norman GR. Mental representations of medical diagnostic knowledge: a review. Acad Med 1996;71(Suppl):24-6.

39. Flexner A. Medical Education in the United States and Canada. A Report to the Carnegie Foundation for the Advancement of Teaching. Boston: Carnegie Foundation for the Advancement of Teaching 1910.

40. Schmidt HG, Norman GR, Boshuizen HP. A cognitive perspective on medical expertise: theory and implication. Acad Med 1990;65:611-621.

41. Woods NN, Brooks LR, Norman GR. The value of basic science in clinical diagnosis: creating coherence among signs and symptoms. Med Educ 2005;39:10712

42. McKenzie CRM. Taking into account the strength of an alternative hypothesis. J Exp Psychol Learn Mem Cogn 1998;24:771-92.

43. Hatala RM, Brooks LR, Norman GR. Practice makes perfect: the critical role of mixed practice in the acquisition of ECG interpretation skills. Adv Health Sci Educ 2003;8:17-26.

44. Thompson L, Gentner D, Loewenstein J. Avoiding missed opportunities in managerial life: analogical learning improves case-based transfer. Organ Behav Human Decis Process 2000;47:98-123.

45. Norman GR, Brooks LR, Rosenthal D, Allen SW, Muzzin LJ. The development of expertise in dermatology. Arch Dermatol 1989;125:1063-68.

46. Nendaz MR, Tekian A. Assessment in problem-based learning medical schools: a literature review. Teach Learn Med 1999;11:232-43.

47. Swanson D, Norman GR, Linn RI. Performance-based assessment: lessons from health professions. Educ Res 1995;24: -12.

48. Eva, KW What every teacher needs to know about clinical reasoning. Med Educ 2005;39:98-106. 